REVISTA DE LA ESCUELA DE CIENCIAS DE LA EDUCACIÓN, AÑo 16, NRO. 15, VOL. 1, ENERO A JUNIO DE 2020. PÁGINAS 1-9. ISSN 2362-3349 (EN LÍNEA). TEXTOS Y VOCES DE LAS POLÍTICAS PÚBLICAS. EL PROGRAMA CONECTAR IGUALDAD (PCI) EN ARGENTINA A PARTIR DE LA NORMATIVA Y SUS DECISORES. BRAIAN MARCHETTI. LUIS PORTA.

\title{
TEXTOS Y VOCES DE LAS POLÍTICAS PÚBLICAS EL PROGRAMA CONECTAR IGUALDAD (PCI) EN ARGENTINA A PARTIR DE LA NORMATIVA Y SUS DECISORES
}

\author{
Braian Marchetti* \\ Universidad Nacional de Mar del Plata - CONICET, Argentina \\ bmarchetti89@gmail.com \\ Luis Porta** \\ Universidad Nacional de Mar del Plata - CONICET, Argentina \\ luisporta510@gmail.com
}

Recibido: 3/06/2019 Aceptado: 6/12/2019

\begin{abstract}
Resumen
Las políticas públicas educativas en materia de inclusión digital conforman un campo estudio vigente al calor de la presencia cada día más fuerte de los medios digitales en nuestra sociedad a partir del incremento de las acciones cotidianas mediadas por tecnologías y del protagonismo de estas en la comunicación y la producción de conocimiento. En Argentina, el Programa Conectar Igualdad significó una bisagra en términos de la magnitud de la inversión económica, del despliegue tecnológico y logístico realizado, y de la transformación de las prácticas educativas institucionales y pedagógicas.

Sobre la base de una perspectiva de estudio que se propone abordar las políticas públicas a través de los relatos y vivencias de los sujetos que las protagonizaron, pretendemos indagar el proceso de creación del PCl desde la exploración del texto de su decreto de creación junto con relatos de los decisores que formaron parte de aquella etapa.
\end{abstract}

Palabras clave: Políticas públicas - Texto de la política - Voces de los sujetos - Conectar igualdad Decisores.

\begin{abstract}
Educational public policies on digital inclusion constitute a field of study in the heat of the increasingly strong presence of digital media in our society, based on the increase of technology use in daily actions and its prominence in communication and. construction of knowledge. In Argentina, the Programa Conectar Igualdad (Connection for Everyone Program) meant a before and after in terms of technology use in Argentinian public education. This was due to the huge investment done in the program, the technological and logistical deployment that was carried out, and the transformation of institutional and pedagogical educational practices it enhanced.

\footnotetext{
* Profesor y Licenciado en Historia (UNMdP). Becario interno doctoral de CONICET. Miembro del Grupo de Investigación en Didáctica de la Historia y Ciencias Sociales (GIEDHICS) perteneciente al Centro de Investigaciones Multidisciplinarias en Educación (CIMED) de la UNMdP. Doctorando en Humanidades y Artes mención Ciencias de la Educación de la UNR. ** Doctor en Filosofía y Ciencias de la Educación. Docente e Investigador (categoría I) - Facultad de Humanidades Centro de Investigaciones Multidisciplinarias en Educación (CIMEd- UNMdP). Director de la Especialización en Docencia Universitaria (UNMdP) y del "Grupo de Investigaciones en Educación y Estudios Culturales" (GIEEC- UNMdP).
} (UNMdP) e Investigador Independiente de CONICET. Profesor Titular Regular Cátedra Problemática Educativa. Director del
\end{abstract}


ReVISTA de LA Escuela de Ciencias de LA EduCACIÓN, AÑo 16, NRO. 15, VOL. 1, ENERO A JUNIO DE 2020. PÁGINAS 1-9. ISSN 2362-3349 (EN LÍNEA). TEXTOS Y VOCES DE LAS POLÍTICAS PÚBLICAS. EL PROGRAMA CONECTAR IGUALDAD (PCI) EN ARGENTINA A PARTIR DE LA NORMATIVA Y SUS DECISORES. BRAIAN MARCHETTI. LUIS PORTA.

We intend to investigate the process of creating the $\mathrm{PCl}$ exploring the text together with its purpose and the stories that the people who was enrolled in the decisive processes that the program went through. We will also take into account the foundations of a study perspective whose aim is to address public policies together with stories and experiences of the subjects who starred it.

Keywords: Public Policies - Text of the Policy - Voices of the Subjects - Connect Equality - Decision Makers.

\section{Introducción}

En América Latina se ha producido en las últimas décadas la expansión de modelos de inclusión tecnológica en el campo educativo, sobre la base de políticas públicas nacionales centradas en iniciativas destinadas al equipamiento de las instituciones, la alfabetización digital y la formación docente. En el caso argentino, el desarrollo del Programa Conectar Igualdad (en adelante PCl) expresa el sentido de estas políticas orientadas desde el Estado nacional para reducir la brecha digital y fomentar la inclusión de tecnologías en el ámbito educativo.

Las dimensiones del PCl y las transformaciones que su puesta en acto ha tenido, generaron para el campo de la investigación en educación múltiples líneas de abordaje que han dado como resultado un vasto espectro de estudios. En el marco de nuestra investigación ${ }^{1}$ para el Doctorado de Humanidades y Artes con Mención en Ciencias de la Educación de la Universidad Nacional de Rosario -la cual se propone comprender los alcances de las políticas públicas de formación docente en inclusión de medios digitales durante el período 2011-2015 a partir del plan de capacitación docente "Escuelas de Innovación"² -, realizamos un análisis sobre el PCl para adentrarnos en su puesta en acto a través de los textos normativos y las voces de los sujetos protagonistas, en términos de haber sido el programa que dio impulso a distintas iniciativas de formación en inclusión digital.

Desde una perspectiva de indagación narrativa característica de los estudios llevados adelante desde el Centro de Investigaciones Multidisciplinarias en Educación de la Universidad Nacional de Mar del Plata, nos proponemos interpretar una política pública a través de los relatos de quienes formaron parte de la construcción y/o interpretación de los textos de la política y participaron activamente en su desarrollo y funcionamiento. Reflexionar entonces sobre la relación entre perspectivas que comprenden a la política como texto y el texto de la política propiamente dicho a través de la experiencia del PCl, permitirá adentrarnos en el centro de la puesta en acto de una política pública de inclusión digital de relevancia continental.

En relación con los objetivos propuestos, cabe aclarar que no resulta de nuestro interés realizar un análisis de la interpretación que se hizo de la puesta en acto del PCl y su llegada a los establecimientos educativos o de analizar la caracterización sobre el programa por parte de los docentes o cada institución educativa. Mediante el análisis documental y la realización de entrevistas en profundidad pretendemos indagar sobre el texto del $\mathrm{PCl}$ a través de su decreto de creación para reflexionar a partir de los relatos de decisores parte de la gestión del PCl sobre lo que permitió hacer, pero al mismo tiempo de las dificultades que implicaron su desarrollo y su construcción posterior en una política de Estado de inclusión digital.

\section{Marco teórico: La política como texto y el texto de la política}

Las políticas públicas desplegadas en materia educativa continúan siendo objeto de debate y análisis según la perspectiva desde la cual se las aborde y la concepción desde la cual se intente reflexionar sobre ellas. Investigadores del campo de la sociología, la ciencia política y las ciencias de la educación forman parte de la construcción del campo de estudio de la política educacional en el que converge una multiplicidad de modos de abordajes y enfoques disciplinarios. En este marco, una línea de estudios ha sido representada por aquellas indagaciones que problematizan sobre la relación entre la sanción de determinadas leyes o normativas y su puesta en práctica y desarrollo dentro del sistema educativo.

Los estudios de Ball (1993; 2012) por ejemplo, se han centrado fundamentalmente en el intento por abordar dos grandes temas: por un lado, la cuestión de la conceptualización de las políticas educativas y, por

\footnotetext{
${ }^{1}$ Título del proyecto de investigación: "Las políticas públicas de formación docente continua en inclusión de medios digitales en Argentina. Un estudio interpretativo sobre la enseñanza de las Ciencias sociales: el caso del plan "Escuelas de Innovación" (2011-2015)".

${ }^{2}$ Destinado a profesores y equipos directivos de escuelas secundarias públicas, tuvo entre sus líneas de acción la realización de acciones de formación presencial y a distancia, con los objetivos de fomentar y mejorar el uso de tecnologías en las prácticas de enseñanza y la gestión institucional, y ofrecer propuestas documentadas de enseñanza y gestión para su uso en todas las escuelas del país. Esta experiencia de formación docente en ejercicio tuvo carácter nacional y abordó la construcción de sus encuentros presenciales en las distintas escuelas del país desde una perspectiva disciplinar de acuerdo a las distintas áreas temáticas.
} 
ReVISTA de LA Escuela de Ciencias de LA EduCACIÓN, AÑo 16, NRO. 15, VOL. 1, ENERO A JUNIO DE 2020. PÁGINAS 1-9. ISSN 2362-3349 (EN LÍNEA). TEXTOS Y VOCES DE LAS POLITICAS PÚBLICAS. EL PROGRAMA CONECTAR IGUALDAD (PCI) EN ARGENTINA A PARTIR DE LA NORMATIVA Y SUS DECISORES. BRAIAN MARCHETTI. LUIS PORTA.

el otro, la indagación sobre las formas de investigar las políticas educativas, sus trayectorias y efectos. Ball ha apuntado a desmantelar críticamente el reduccionismo implícito de los estudios que no comprenden ni documentan cómo las escuelas lidian con las políticas que "ingresan" a sus edificios ni cómo sus actores transforman creativamente las ideas y los textos de las políticas (resoluciones, leyes, decretos, memos, manuales, formularios, registros de información, etc.) en prácticas en contextos y realidades específicas. Según él, las políticas deberían ser entendidas como procesos que son, de manera muy heterogénea y constante, sujetos a interpretaciones y se imprimen en las formas creativas en que son puestas en acto (más que "implementadas") en las instituciones y sus aulas (Beech y Meo, 2016).

Al desarrollar esta teoría de la puesta en acto de las políticas y de cómo los actores le otorgan sentido, las median, cuestionan o redefinen y, muchas veces, las marginalizan o desconocen, Ball (2012) considera que es necesario observar las políticas en acción, rastreando cómo fuerzas políticas y sociales, instituciones, personas, eventos, intereses y azar se entrelazan, implicando reconocer la importancia de las relaciones de poder y de los intereses que movilizan a diversos actores. En este sentido, la puesta en acto de la política siempre supone la traducción de los textos de la política en acciones y de las formulaciones abstractas contenidas en las ideas de las políticas en prácticas situadas. En la misma sintonía se encuentran los planteos de Sautu (2010), para quien el "texto", es decir, la concepción y el diseño de una política es resignificado por múltiples actores individuales y colectivos que participan de los procesos tanto de revisión de su diseño cómo de su implementación, la cual será "construida" por los actores sociales que participen en los distintos niveles de desarrollo del programa educativo.

Al reflexionar sobre el modo en que los profesores de escuelas secundarias perciben las políticas educativas, Yuni (2018) sostiene que aquello que se instituye como el texto de las políticas públicas en educación es producto de un proceso de disputa y de confrontación entre diferentes sectores que pretenden imponer su propia cosmovisión, por lo que para comprender las marcas subjetivantes de las políticas es necesario considerar su ciclo. Es decir, desde el momento de su formulación hasta que estas se encarnan en los sujetos, son reconstruidas y recreadas por ellos en relación con el contexto y con los demás sujetos que intervienen, y son llevadas del texto a la práctica.

De esta manera, las políticas educativas resultan construcciones en múltiples niveles que deben ser analizadas como procesos dentro de un contexto macropolítico e institucional. Braun, Ball, Maguire y Hoskins (2017) prestan especial atención al contexto de puesta en acto de las políticas educativas al incorporar el estudio sobre las condiciones objetivas vinculadas a un conjunto de dinámicas subjetivas de interpretación. Esta acción los conduce a reconocer que lo material, estructural y relacional deben ser considerados como parte del análisis de políticas y que el estudio de los contextos situados, profesionales, materiales y externos romperá con el idealismo tradicional que piensa la implementación de las políticas educativas a partir de escenarios ideales.

Por otro lado, además de la centralidad otorgada a los sujetos que interpretan los textos de las políticas, vale mencionar el lugar que Ball (2002) le otorga a los autores de los textos ya que considera que si bien estos no pueden controlar el significado de sus textos, realizan los esfuerzos necesarios para afirmar tal control con los medios disponibles y lograr una "correcta" lectura (Ball, 2002). En ese marco, resulta relevante comprender aquellos esfuerzos y sus efectos sobre los lectores para reconocer la atención que éstos prestan al contexto de producción e intención comunicativa de los que escriben.

En relación con el análisis documental y al pensar en cómo abordar textos institucionales, Rockwell (2015) propone además de indagar sobre lo escrito imaginar sobre lo no documentado. Para realizar este planteo, retoma una obra de Julia (1995) donde para estudiar una cultura escolar diversa y cambiante el autor sugiere tender puentes entre los documentos normativos y la práctica educativa a través de una lectura de los documentos que den cuenta del entrelazamiento y la distancia entre las normas educativas y las prácticas culturales en las escuelas. Algunas maneras de construir este entrelazamiento son prestando atención a las omisiones dentro de los documentos oficiales infiriendo la presencia de determinadas prácticas a partir de las proscripciones o prescripciones, indagando sobre las condiciones de materialidad de los documentos, o analizando los discursos oficiales y su vinculación con la normativa.

En definitiva, si retomamos los distintos aportes de los autores mencionados en líneas anteriores podemos afirmar nuestra concepción de la puesta en acto de las políticas en la síntesis que realiza Ball (2002) al plantear que comprender a la política como texto "implica pensar en representaciones que son codificadas vía disputas, compromisos, interpretaciones y reinterpretaciones de la autoridad pública, y decodificadas vía interpretaciones y significados de los actores según sus historias, experiencias, habilidades, recursos y contexto" (Ball, 2002, p.21). Esta complejidad que otorga los distintos momentos que atraviesa el texto de la política hasta convertirse en una proclama oficial y luego para ser llevado a la práctica en los ámbitos educativos pone una especial centralidad en los sujetos que protagonizan las distintas instancias mencionadas. Siguiendo a Bohoslavsky y Soprano (2010) podemos concebir a estos sujetos como quienes representan el "rostro humano" del Estado, por lo que se convierte imprescindible superar a las perspectivas más estructurales y normativas de acercamiento al Estado y reparar en las prácticas cotidianas, los diversos ámbitos de interlocución, y en la multiplicidad de contactos sociales en los que participan quienes "son" el Estado. 
Revista de LA Escuela de Ciencias de LA EduCACIÓn, AÑo 16, NRO. 15, VOL. 1, ENERO A JUNIO de 2020. PÁGINAS 1-9. ISSN 2362-3349 (EN LíneA). TEXTOS Y VOCES DE LAS POLÍTICAS PÚBLICAS. EL PROGRAMA CONECTAR IGUALDAD (PCI) EN ARGENTINA A PARTIR DE LA NORMATIVA Y SUS DECISORES. BRAIAN MARCHETTI. LUIS PORTA.

\section{Marco metodológico}

Como hemos mencionado en la introducción, este trabajo se enmarca en un proyecto de investigación mayor el cual se ubica dentro del campo de la investigación cualitativa pretendiendo comprender los fenómenos sociales desde su escenario natural y a través de los significados que las personas les dan (Denzin y Lincoln, 2011). De esta manera, se buscará interpretar las políticas públicas de formación docente continua en inclusión de tecnologías digitales para la enseñanza de las Ciencias Sociales en nivel secundario desde el plan Escuelas de Innovación, a través de la recolección de materiales empíricos y la utilización de una amplia gama de prácticas interpretativas interconectadas entre sí.

Las fuentes de información utilizadas para este trabajo podemos dividirlas en dos grupos: por un lado, los documentos escritos y publicados por distintos órganos de gobierno, y por el otro, los decisores de política pública intervinientes en el PCl. Teniendo en cuenta que la mayor parte de los registros oficiales y documentos públicos son de fácil acceso, en este caso se trabajará con documentos como la Ley Nacional de Educación, el decreto de creación del PCl y resoluciones del Consejo Federal de Educación poniendo el foco de atención no sólo en lo que dicen los textos sino también en los silencios u omisiones que estos presentan y los modos en que se estructuran y organizan los problemas y planteos abordados (Rapley, 2007).

En referencia a los decisores, formarán parte de nuestro desarrollo argumentativo fragmentos de cuatro entrevistas en profundidad (Taylor y Bogdan, 1990). Los entrevistados han sido elegidos por su relevancia en la toma de decisiones dentro de las estructuras del Ministerio de Educación de la Nación y de ANSES/PCI. El Decisor 1 tuvo a su cargo la coordinación de un área de trabajo vinculada a la inclusión digital en el Ministerio de Educación (D1) y el Decisor 2 fue un alto funcionario dentro del Ministerio (D2), mientras que los Decisores 3 y 4 formaron parte de la estructura de ANSES dedicada al PCl cumpliendo roles de dirección (D3) y de coordinación logística (D4). Vale aclarar que la selección de la numeración responde al orden de aparición en el trabajo y no refleja jerarquías ni clasificaciones cualitativas.

Desde nuestra perspectiva, analizar una política pública a través de los sujetos que la protagonizaron implica retomar sus voces y prácticas para adentrarnos en la experiencia de los procesos de diseño y puesta en acto del programa. Para ello adoptamos el enfoque narrativo, en donde sus relatos darán lugar al acercamiento a las dinámicas y dimensiones personales, para construir un relato mayor que permita interpretar nuevas historias sobre los cuales inscribir el posible cambio o mejora (Bolívar, Domínguez y Fernández, 2001).

\section{El texto del Conectar Igualdad}

La Ley de Educación Nacional № 26.206 sancionada en el año 2006 en Argentina, incorpora como parte de los fines y objetivos de la política educativa nacional al desarrollo de las competencias necesarias para el manejo de los nuevos lenguajes producidos por las tecnologías de la información y la comunicación. Al mismo tiempo, como parte de las políticas de promoción de la igualdad educativa y de la calidad de la educación, se propone desarrollar opciones educativas basadas en el uso de las tecnologías y de los medios masivos que colaboren con el cumplimiento de los fines y objetivos de la ley. En este marco, el Programa Conectar Igualdad fue lanzado en el año 2010 a través del decreto presidencial № 459/10 como política pública de inclusión digital educativa. Este programa estuvo destinado a garantizar el acceso y uso de las tecnologías y a mejorar los procesos de enseñanza y de aprendizaje a través de la modificación de las formas de trabajo en el aula y en la escuela a partir del modelo 1 a 1, el cual involucra a un estudiante, una computadora, una experiencia de aprendizaje personalizada y un contexto de conexión inalámbrica con acceso a internet (Manso, 2011).

EI PCl contempló la distribución de netbooks a todos los alumnos y docentes de la educación Secundaria, de la Educación Especial y de los Institutos Superiores de Formación Docente pertenecientes al sistema de gestión estatal, así como también se ocupó de proveer un piso tecnológico a todas los establecimientos educativos afectados. Según datos del informe de "Política Educativa Nacional 2003-2015" elaborado por el Ministerio de Educación de la Nación, para agosto de 2015 ya habían sido entregadas más de 5 millones de notebooks alcanzando los 11.000 establecimientos educativos e impactando positivamente en la disminución de la brecha digital. De acuerdo a este informe, el programa contribuyó a su reducción habiendo un $29 \%$ de los estudiantes para los cuales la notebook del PCl fue la primera computadora del hogar, ascendiendo este número al $43 \%$ para el caso de contextos rurales y al $47 \%$ para contextos de vulnerabilidad socioeconómica.

Más allá de cualquier apreciación sobre el programa, cuyo análisis no forma parte de los objetivos de este trabajo, resulta innegable considerar la relevancia que tuvo en cuanto a su envergadura en términos de inversión económica, tecnológica, logística, y de transformación de las prácticas educativas cotidianas institucionales y pedagógicas. Una muestra de ello lo representan las distintas líneas de investigación que se 
ReVISTA de LA Escuela de Ciencias de LA EduCACIÓN, AÑo 16, NRO. 15, VOL. 1, ENERO A JUNIO DE 2020. PÁGINAS 1-9. ISSN 2362-3349 (EN LÍNEA). TEXTOS Y VOCES DE LAS POLÍTICAS PÚBLICAS. EL PROGRAMA CONECTAR IGUALDAD (PCI) EN ARGENTINA A PARTIR DE LA NORMATIVA Y SUS DECISORES. BRAIAN MARCHETTI. LUIS PORTA.

desplegaron desde ámbitos académicos y gubernamentales ${ }^{3}$, abocadas a la medición y elaboración de estadísticas, a la definición del impacto o las transformaciones a través del análisis de percepciones, perspectivas y desafíos surgidos en las instituciones escolares, directivos, estudiantes y docentes a partir de la entrega de las netbooks, a la implementación en distintas provincias, y al estudio de la incidencia del programa en la relación pedagógica y sobre los niveles de desigualdad social constituyen distintas líneas dentro las cuales variados proyectos de investigación han abordado al Conectar Igualdad y su desarrollo. Por lo tanto, analizar el texto que le dio origen a esta política de inclusión digital entrecruzando su articulado con las apreciaciones y perspectivas de los sujetos que formaron parte de su diseño y puesta en acto, resulta de especial interés al abordar al Programa desde una perspectiva que se encuentra en vacancia.

Como mencionamos en líneas anteriores, el PCl tuvo su inicio con la sanción del decreto presidencial $N^{\circ}$ 459/10 con fecha del 6 de abril de 2010 en donde se explicita "Créase el "Programa Conectar Igualdad.Com.Ar" de incorporación de la nueva tecnología para el aprendizaje de alumnos y docentes". Con una extensión de tres páginas, el decreto se encuentra organizado en dos secciones separadas por los considerandos y el articulado correspondiente. En sus considerandos el decreto establece un marco ideológico al desarrollar la concepción desde la cual comprende la relación entre educación y Estado. Consagrar el derecho de enseñar y de aprender, comprender a la educación como un derecho personal y social garantizado por el Estado, y pretender que la escuela forme sujetos responsables capaces de utilizar el conocimiento como herramienta para comprender y transformar su entorno, son los principales postulados que dan cuenta de la perspectiva que sustenta el PCl.

Partiendo de la vocación por garantizar el derecho a la igualdad, dentro de los considerandos se reconocen las transformaciones que los medios digitales han generado en la sociedad moderna y se advierte sobre la necesidad de producir respuestas al respecto por parte del Estado. En ese marco, en los distintos párrafos que componen la sección inicial del decreto se argumenta sobre la necesidad de crear un programa de incorporación de tecnologías para el aprendizaje en línea y red, proporcionando a alumnas, alumnos y docentes de la educación pública secundaria y especial una computadora. Asimismo, el escrito señala a la capacitación docente como una de las tareas a realizar para lograr el acompañamiento a los docentes en el uso de las computadoras entregadas.

En la sección que comprende los once artículos del decreto se establecen los lineamientos principales del $\mathrm{PCl}$ explicitando los objetivos principales del programa y su estructura de funcionamiento y presupuestaria. En sintonía con el contexto establecido en sus considerandos el articulado comienza con la definición de la base central del programa:

Créase el "PROGRAMA CONECTAR IGUALDAD.COM.AR" con el fin de proporcionar una computadora a alumnas, alumnos y docentes de educación secundaria de escuelas públicas, de educación especial y de Institutos de Formación Docente, capacitar a los docentes en el uso de dicha herramienta y elaborar propuestas educativas con el objeto de favorecer la incorporación de las mismas en los procesos de enseñanza y de aprendizaje. (Art. $N^{\circ} 1$. Decreto $N^{\circ} 459 / 10$ )

Sin dar ningún tipo de especificidad técnica o de modalidad de entrega, en este artículo la esencia del programa queda establecida al indicar el formato del modelo 1 a 1 como propuesta de inclusión digital y seleccionar a estudiantes y docentes de distintas modalidades y niveles como los receptores de las computadoras. La necesidad de generar instancias de capacitación docente se convierte en el otro pilar central que acompaña la entrega de computadoras reconociendo una perspectiva pedagógica que comprende a la enseñanza y el aprendizaje como dos procesos interrelacionados pero distintos donde el desarrollo de uno no implica necesariamente como consecuencia inmediata el desarrollo del otro.

Los cinco artículos siguientes hacen referencia a la estructura jerárquica del programa, sus integrantes y deberes y funciones. La primera resolución al respecto sanciona la creación del Comité Ejecutivo del PCI, estableciendo la presidencia para el Director Ejecutivo de la Administración Nacional de la Seguridad Social (ANSES) y delineando su composición con un representante de la Jefatura de Gabinete de Ministros, un representante del Ministerio de Educación, un representante del Ministerio de Planificación Federal, Inversión Publica y Servicios, y un representante de la Administración Nacional de la Seguridad Social. Más adelante en el tiempo y, con el correr de la realización de las entrevistas en profundidad, pudimos vislumbrar que esta conformación basada en la articulación entre distintos estamentos estatales supuso un componente interministerial que implicaría luego para el funcionamiento del Programa la necesidad de la

\footnotetext{
${ }^{3}$ Un resumen en Marchetti, B. (2018) "El Programa Conectar Igualdad y sus líneas de investigación. Una propuesta de estudio sobre la formación docente continua desde la Didáctica de las Ciencias Sociales". En Jara, M. (comp.) Los aportes de la didáctica de las ciencias sociales, de la historia y de la geografía a la formación de la ciudadanía en los contextos iberoamericanos. Actas del XVII Jornadas Nacionales y VI Internacionales de Enseñanza de la Historia y IV Encuentro Iberoamericano de Investigación en Didáctica de las Ciencias Sociales. APEHUN. ISBN 978-987-42-9448-7
} 
ReVISTA de LA Escuela de Ciencias de LA EduCACIÓN, AÑo 16, NRO. 15, VOL. 1, ENERO A JUNIO DE 2020. PÁGINAS 1-9. ISSN 2362-3349 (EN LÍNEA). TEXTOS Y VOCES DE LAS POLÍTICAS PÚBLICAS. EL PROGRAMA CONECTAR IGUALDAD (PCI) EN ARGENTINA A PARTIR DE LA NORMATIVA Y SUS DECISORES. BRAIAN MARCHETTI. LUIS PORTA.

construcción de acuerdos y consensos hacia el interior del Comité, así como la presencia de fuertes tensiones y problemáticas.

En lo que refiere a la reglamentación de este Comité, el decreto establece que el mismo Comité lo dicte, pero deja en claro su misión principal:

El Comité Ejecutivo tendrá por misión determinar las condiciones para ejecutar el Programa, y deberá proponer las acciones a efectos de lograr todos los objetivos del mismo, quedando a su cargo el dictado de normas complementarias y su implementación técnica y operativa. (Art. N4. Decreto $\left.\mathrm{N}^{\circ} 459 / 10\right)$

A partir de aquí queda en claro la centralidad otorgada al Comité Ejecutivo y la relevancia de su puesta en marcha y desarrollo para el funcionamiento del Programa, ya que tanto su reglamentación interna como la generación de disposiciones necesarias para garantizar el cumplimiento de los objetivos del $\mathrm{PCl}$ quedaron en manos del mismo Comité. En su interior no sólo se discutirían las cuestiones referidas a adquisición de partidas presupuestarias, licitaciones, selección de hardware y software, planificación de la distribución de computadoras y del tendido de fibra óptica, sino que también entraría en juego una fuerte matriz política como se establece en el artículo $N^{\circ} 6$, donde se le delega la responsabilidad al Comité de promover "los acuerdos necesarios con las Provincias y la Ciudad Autónoma de Buenos Aires para el adecuado cumplimiento de los objetivos del Programa".

En relación con las disposiciones presupuestarias, las mismas comprenden los artículos № 8,9 y 10 . En ellos se establece la creación dentro de ANSES de un programa sustentado en partidas presupuestarias del Presupuesto Nacional mientras que el dictado de la normativa que regule dicha asistencia financiera queda bajo la responsabilidad de ANSES. La realización de las adecuaciones presupuestarias necesarias para ejecutar la medida queda en manos del Jefe de Gabinete de Ministros.

La sanción de este decreto conforma un acontecimiento bisagra en cuanto a las políticas públicas de inclusión digital en Argentina. Las características particulares que el mismo posee pueden resumirse en establecer los objetivos generales del programa, el órgano de ejecución del mismo y su sustento presupuestario. Si bien el decreto no deja especificidades técnicas en relación a cuestiones de índole operativa en términos del tipo de equipamiento, logística o distribución deja esas tareas en manos del Comité Ejecutivo. Por último, resulta interesante advertir el espíritu de colaboración interministerial que se propone al crear un Comité compuesto por distintos organismos del Estado y de cuya articulación dependió posteriormente el funcionamiento general del PCl.

\section{Las voces del texto}

Una de las características que señala Ball (2002) sobre la construcción de los textos de la política pública es su producción a través de distintas etapas que implican tensiones, acuerdos y modificaciones en el escrito original. De esta manera, pueden suceder modificaciones al escrito original en las micropolíticas de la formulación legislativa, en el proceso parlamentario y/o en las políticas e intereses de los distintos actores y grupos de gobierno. En el caso del $\mathrm{PCl}$, su decreto de creación no fue ajeno a este proceso en donde se produjo una articulación entre el Ministerio de Educación de la Nación y Presidencia de la Nación que incluyó modificaciones en su publicación final.

EI PCl tuvo un antecedente inmediato en un programa denominado Programa de Inclusión Digital Educativa (PIDE) que se implementó durante 2009 y 2010 entregando computadoras en escuelas técnicas. A partir de allí comenzó a gestarse la primera propuesta que se construyó desde el Ministerio y fue elevado para su aprobación. La buena experiencia del PIDE impulsó la búsqueda de caminos posibles desde el Ministerio de Educación para ampliar el programa hacia otros niveles. Al ser consultado uno de los entrevistados sobre el proceso que dio origen al PCl cuenta sobre la relevancia que tomó dentro del gobierno la inclusión digital y señala que a partir del PIDE comenzó un intercambio con el Poder Ejecutivo nacional en el cual según "la Presidenta estaba muy interesada en ampliar el programa y ella decidió que fuera sobre secundaria en general" (D1). Desde el Ministerio consideraban de gran importancia el "costado social" que tenía que tener el programa, así como la modalidad de educación especial y la formación docente.

La figura de la Presidenta comienza a aparecer en los relatos de los entrevistados con un rol activo e interviniendo en el delineamiento del futuro programa. Tal es así que este intercambio entre el Ministerio de Educación y Presidencia terminó por definir los lineamientos fundamentales de lo que sería luego el PCl. De esta manera, el Ministerio elaboró un proyecto inicial al cual le habría sido dotado de su estructura fundamental dentro del ámbito de Presidencia:

Presentamos la iniciativa a la Presidenta, que luego de pensarlo tomó la brillante decisión de construir una política mucho más ambiciosa, articulada con otras dependencias del Estado Nacional: ANSES compraba y distribuía, Ministerio de Educación aportaba la mirada pedagógica y capacitaba a los docentes y el Ministerio de Planificación Federal se dedicaba a generar instalaciones, pisos tecnológicos e internet en las escuelas. (D2) 
ReVISTA de LA Escuela de Ciencias de LA EduCACIÓN, AÑo 16, NRO. 15, VOL. 1, ENERO A JUNIO DE 2020. PÁGINAS 1-9. ISSN 2362-3349 (EN LÍNEA). TEXTOS Y VOCES DE LAS POLÍTICAS PÚBLICAS. EL PROGRAMA CONECTAR IGUALDAD (PCI) EN ARGENTINA A PARTIR DE LA NORMATIVA Y SUS DECISORES. BRAIAN MARCHETTI. LUIS PORTA.

Este formato final del proyecto se estructuró no sin generar ciertas disconformidades en algunos de los miembros del Ministerio. Una de las principales modificaciones realizadas al proyecto de decreto inicial tuvo que ver con la introducción de ANSES como cabeza del programa. En referencia a esta cuestión, uno de los entrevistados comenta que el proyecto "no había salido así, para nosotros era un proyecto educativo, pero sí lo habíamos pensado desde el principio como algo interministerial donde había fuerte participación de todos y el Comité funcionaba bien" (D1). En este caso, podríamos señalar que, si bien desde el Ministerio no cuestionaron la articulación de trabajo con otras áreas de gobierno, la centralidad de otros organismos dentro del proyecto fue causante de tensiones hacia el adentro de la gestión.

Por otro lado, al analizar el texto del decreto en apartados anteriores sosteníamos que, si bien definía claramente los objetivos del programa y delineaba los esquemas básicos de su estructura organizativa, se encontraban ausentes algunas definiciones de relevancia. Al ser consultado por qué debilidades podría señalar en relación con el $\mathrm{PCl}$, uno de los entrevistados reconoce que por lo general los decretos que crean este tipo de programas "son escuetos" (D3) pero considera que el decreto del PCl podría haber contenido directivas más precisas:

El decreto que crea Conectar Igualdad dice pocas cosas, dice que hay que entregarle una computadora a cada estudiante y docente de la enseñanza media de gestión pública, así como a los institutos de formación docente y a las escuelas especiales, no decía mucho más que eso. (D3)

Al reflexionar sobre situaciones prácticas que se hayan complejizado por la falta de ciertas precisiones en la normativa, el entrevistado señaló distintas situaciones que se presentaron en la práctica y que tuvieron que resolver como por ejemplo "qué tipo de computadora había que entregar y quiénes eran los beneficiarios".

En el mismo sentido, otro de los entrevistados caracteriza al decreto como escrito "económicamente" (D4) y para él haber tenido que completar ese texto con políticas públicas a partir de su interpretación condujo a una situación forzada. Su planteo consiste en considerar que el decreto "podría haber sido más preciso en la formulación del universo de los titulares del nuevo derecho, como así también en establecer claramente el objetivo de desarrollo industrial, es decir, participación de la industria de micro informática, software y contenidos didácticos" (D4).

Retomando el análisis de Ball (2002) las políticas crean las circunstancias para que el margen de opciones disponibles para decidir sea lo más estrecho posible a través de metas o resultados particulares pero que no deja de resultar cambiante. En ese marco, con respecto a los destinatarios del $\mathrm{PCl}$ el decreto establece a "alumnas, alumnos y docentes de educación secundaria de escuelas públicas, de educación especial y de Institutos de Formación Docente" (Decreto $\left.N^{\circ} 459 / 10\right)$. Sin embargo, dos de los decisores entrevistados (D3 y D4) señalan que la cuestión de los beneficiarios conformó uno de los vacíos que complejizaron la puesta en acto del programa.

La complejidad del sistema educativo que contiene escuelas secundarias que dependen de universidades, las escuelas secundarias que dependen de los municipios o el caso de estudiantes que se encuentran no institucionalizados donde por accidentes o enfermedades de largo tratamiento se encuentran fuera de la institución educativa, son algunos de los ejemplos mencionados de situaciones no especificadas en el decreto que tuvieron que resolverse en pleno desarrollo del programa. De la misma manera, la falta de sistematización de la información en relación con los establecimientos educativos generó complicaciones al momento de relevar los datos de los beneficiarios:

Uno se preguntaba quién tiene la lista de los alumnos y los profesores, entonces en el Ministerio de Educación no sabían quiénes eran, cuando empecé a preguntar en las provincias en general tampoco, así que hubo que resolver todos esos desafíos sobre la marcha y llevar el programa adelante. (D3)

Por último, más allá de estas situaciones es necesario señalar que posteriormente el decreto del PCl contó con el acompañamiento de la resolución del Consejo Federal de Educación $N^{\circ} 123 / 10$ que contiene dentro de sus anexos al documento "Políticas de Inclusión Digital Educativa del Programa Conectar Igualdad" (Anexo I) y al "Manual operativo para la gestión institucional del Programa Conectar Igualdad" (Anexo II). Esta resolución fue publicada en diciembre de 2010, seis meses después de la promulgación del decreto de creación del $\mathrm{PCl}$ y se presenta como el resultado del trabajo hacia el interior del Consejo Federal por avanzar en políticas de integración digital, así como de los esfuerzos de articulación realizados por el Comité Ejecutivo del PCl. Dentro de sus considerandos contempla que "entre el Comité Ejecutivo del Programa Conectar igualdad y cada gobierno jurisdiccional se firmaron convenios marco de adhesión que establecen las pautas generales y responsabilidades para la ejecución de dicho programa" (Res. CFE N¹23/10).

Los Anexos I y II de dicha resolución describen los principios orientadores y las líneas estratégicas de la implementación del Programa. Dentro de ellos se definen sus finalidades, sus objetivos generales, el enfoque pedagógico, la estrategia educativa, la modalidad de adquisición del equipamiento y sus variantes 
ReVISTA de LA Escuela de Ciencias de LA EduCACIÓN, AÑo 16, NRO. 15, VOL. 1, ENERO A JUNIO DE 2020. PÁGINAS 1-9. ISSN 2362-3349 (EN LÍNEA). TEXTOS Y VOCES DE LAS POLÍTICAS PÚBLICAS. EL PROGRAMA CONECTAR IGUALDAD (PCI) EN ARGENTINA A PARTIR DE LA NORMATIVA Y SUS DECISORES. BRAIAN MARCHETTI. LUIS PORTA.

según los destinatarios, la organización de la gestión del Programa, y las acciones de seguimiento y la evaluación. De esta manera, consideramos que la resolución aludida buscó atender seis meses después los aspectos a los que hicieron alusión los decisores entrevistados como omisiones que complejizaron el comienzo del funcionamiento del $\mathrm{PCl}$ al no ser explicitados en el decreto de creación.

\section{Algunas conclusiones de esta etapa}

En este trabajo hemos procurado establecer una conexión entre la producción del texto de una política pública y su puesta en acto a partir del caso del PCl. Para ello comenzamos por recuperar los principales aportes teóricos que fundamentan la preocupación por los distintos momentos de construcción de una política desde su planificación inicial hasta su concreción y la interpretación de sus lineamientos que conlleva por parte de quienes lo traducen en la práctica. En segunda instancia, realizamos un recorrido descriptivo sobre el decreto presidencial $\mathrm{N}^{\circ} 459 / 10$ el cual dio origen al PCl, y que nos permitió identificar las bases fundamentales del programa y analizar la composición de su articulado organizado en tres bloques de contenido compuestos por sus objetivos principales, su organización interna y su financiamiento.

Tomando como punto de partida un enfoque que pone de relieve los relatos de los sujetos que protagonizaron la construcción de los documentos normativos y la puesta en marcha de las políticas que aquellos textos sustentan, continuamos el trabajo articulando distintas opiniones vertidas en relación con el texto normativo del PCl y su interacción en la práctica misma del desarrollo del programa, a partir del diálogo con decisores del Ministerio de Educación de la Nación y del sector del PCI ubicado dentro de la estructura de ANSES.

Este recorrido nos ha permitido identificar algunas cuestiones de interés al momento de reflexionar sobre la construcción y puesta en acto de una política pública a partir de la concepción de la política como texto (Ball, 2002) referida en apartados anteriores. En primer lugar, es posible destacar la interacción entre dos espacios de gobierno como Presidencia y el Ministerio de Educación de la Nación al momento de construir el decreto del PCl. Esta articulación además no se dio sin haber generado modificaciones ni tensiones en relación a las modificaciones realizadas. Es decir, la articulación construida entre el Ministerio y Presidencia al momento de pensar y construir un programa de inclusión digital explicita las modificaciones que fueron realizándose sobre la propuesta inicial al darle mayor amplitud y cobertura al programa, así como al definir su estructura de gestión al colocar como cabeza del proyecto a ANSES y dotarlo de un carácter interministerial. Si bien esta lógica de articulación entre áreas de gobierno formó parte de la propuesta inicial del Ministerio, no lo fue así la centralidad otorgada posteriormente a ANSES.

En segundo lugar, los relatos de los entrevistados aluden directamente a otro actor clave en la construcción del decreto como fue "la Presidenta". Tanto para explicitar el diálogo construido entre el Ministerio y la Presidenta como para reconocer o criticar algunas de las modificaciones realizadas al decreto inicial proyectado desde el Ministerio, la figura de la Presidenta aparece recurrentemente como un actor clave en la definición del perfil y característica del PCl.

Por último, la alusión de los entrevistados a lo "escueto" del articulado del decreto y las complicaciones y situaciones complejas por resolver que dejó para la práctica es otra de las cuestiones a señalar. Sin embargo, en este caso resulta interesante rescatar uno de los aportes de Ball (2002) que recupera el contexto en el que "caen" los textos de la política. Es decir, los textos no caen "llovidos del cielo" ni ingresan en un vacío institucional, según el autor quienes redactan el texto y quienes lo interpretan posteriormente se encuentran insertos en un contexto determinado, con una historia representada e interpretada. Esta perspectiva permite entender por qué un decreto "escrito económicamente" como se señaló anteriormente pudo igualmente dar forma e impulso a uno de los programas de inclusión digital más ambiciosos del continente americano.

La normativa inicial del PCl permitió entonces generar un programa de inclusión muy importante que tuvo lugar a pesar de la falta de precisión señalada en ella gracias al contexto en el que se desarrolló y a los actores en los cuales "cayó" ese texto. Más allá de lo general del articulado del decreto, a partir de su interpretación por los sujetos decisores de los ámbitos de gestión del Ministerio de Educación y de ANSES se logró dar inicio a una política de inclusión digital que alcanzó a repartir cinco millones de netbooks, expandir fibra óptica y pisos tecnológicos en establecimientos educativos a lo largo y ancho del país, realizar diversas instancias de capacitación y afrontar los desafíos que surgieron por delante gracias a un contexto de crecimiento del Estado, de ampliación de derechos, de aumento de inversión en educación que enmarcaron el desarrollo del programa.

\section{Referencias bibliográficas}

Ball, S. J. (1993). What is policy? Texts, trajectories and toolboxes. Discourse: Studies in the Cultural Politics of Education, 13 (2), 10-17.

(2002). Textos, discursos y trayectorias de la política: la teoría estratégica. Páginas. Revista de la Escuela de Ciencias de la Educación, 2 y 3 (2), 19-33.

(2012). Global education inc: New policy networks and the neo-liberal imaginary. London: Routledge. 
ReVista de la Escuela de Ciencias de LA EduCACIÓN, AÑo 16, NRO. 15, VOL. 1, ENERO A JUNiO de 2020. PÁGINAS 1-9. ISSN 2362-3349 (EN LínEA). TEXTOS Y VOCES DE LAS POLÍTICAS PÚBLICAS. EL PROGRAMA CONECTAR IGUALDAD (PCI) EN ARGENTINA A PARTIR DE LA NORMATIVA Y SUS DECISORES. BRAIAN MARCHETTI. LUIS PORTA.

Beech, J. y Meo, A. I. (2016). Explorando el uso de las herramientas teóricas de Stephen J., Ball en el estudio de las políticas educativas en América Latina. Archivos Analíticos de Políticas Educativas, 24(23).

Bohoslavsky, E. y Soprano, G. (Eds.) (2010). Un Estado con rostro humano. Funcionarios e instituciones estatales en Argentina (desde 1880 hasta la actualidad). Buenos Aires: Prometeo.

Bolívar, A.; Domínguez J. y Fernández, C. (2001). La investigación biográfico-narrativa en educación. Enfoque y metodología. Madrid: La muralla.

Braun, A.; Ball, S. J.; Maguire, M., y Hoskins, K. (2017). Tomando el contexto escolar seriamente: hacia una explicación de la puesta en práctica de las políticas en la escuela secundaria. En Miranda, E. M., y Lamfri, N. Z. (org.) La educación secundaria. Cuando la política educativa llega a la escuela. Buenos Aires: Miño y Dávila.

Denzin, N. K. y Lincoln, Y. S. (Comps.) (2011). El campo de la investigación cualitativa. Barcelona: Gedisa.

Julia, D. (1995). La cultura escolar como objeto histórico. En: Menegus, M. y Gonzalez, E. (Coords.) Historia de las universidades modernas en Hispanoamérica. México: Universidad Autónoma de México.

Manso, M. (2011). Las TIC en las aulas, experiencias latinoamericanas. Buenos Aires: Paidós.

Rapley, T. (2007). Los análisis de la conversación, del discurso y de documentos en investigación cualitativa. Madrid: Morata.

Rockwell, E. (2015). La Experiencia Etnográfica. Historia y cultura en los procesos educativos. Buenos Aires: Paidós.

Sautu, R. (2010). Sugerencias para el desarrollo de la investigación científica en educación. En Wainerman, C. y Di Virgilio, M. M. (Comps.). El quehacer de la investigación en educación. Buenos Aires: Manantial.

Taylor, S. J. y Bodgan, R. (1990). La investigación cualitativa. Barcelona: Gedisa.

Yuni, J. A. (Dir.) (2018). Inclusión/es en la Escuela Secundaria. Itinerarios de lo posible. Catamarca: Encuentro Grupo Editor.

\section{Documentos oficiales}

Congreso de la Nación Argentina (2006) Ley de Educación Nacional №26.206. Recuperado de http://servicios.infoleg.gob.ar/infolegInternet/anexos/120000-124999/123542/norma.htm

Decreto del PEN (2010) N459/10. Recuperado de http://servicios.infoleg.gob.ar/infoleglnternet/anexos/165000169999/165807/norma.htm

Ministerio de Educación de la Nación (2015) Informe "La política educativa nacional 2003-2015". Buenos Aires. Versión impresa.

Consejo Federal de Educación (2010) Resolución No 123/10. Recuperado de https://cfe.educacion.gob.ar/resoluciones/res10/123-10.pdf 